\title{
Shape-Based Curve Growing Model and Adaptive Regularization for Pulmonary Fissure Segmentation in CT
}

\author{
Jingbin Wang ${ }^{1}$, Margrit Betke ${ }^{1}$, and Jane P. Ko ${ }^{2}$ \\ 1 Computer Science Department, Boston University, Boston MA 02215, USA \\ 2 Department of Radiology, New York University, New York NY 10016, USA \\ \{jingbinw, betke\}@cs.bu.edu, www.cs.bu.edu/groups/ivc
}

\begin{abstract}
This paper presents a shape-based curve-growing algorithm for object recognition in the field of medical imaging. The proposed curve growing process, modeled by a Bayesian network, is influenced by both image data and prior knowledge of the shape of the curve. A maximum a posteriori (MAP) solution is derived using an energy-minimizing mechanism. It is implemented in an adaptive regularization framework that balances the influence of image data and shape prior in estimating the curve, and reflects the causal dependencies in the Bayesian network. The method effectively alleviates over-smoothing, an effect that can occur with other regularization methods. Moreover, the proposed framework also addresses initialization and local minima problems. Robustness and performance of the proposed method are demonstrated by segmentation of pulmonary fissures in computed tomography (CT) images.
\end{abstract}

\section{Introduction}

Enormous demands for automatically recognizing complicated anatomical structures in medical images have been raised in recent years. The medical community has seen many benefits from computer aided diagnosis (CAD) systems [5] and computer visualizations [7]. A large body of literature on segmentation of anatomical structures has been published [14. Low-level image processing methods, for example, thresholding or edge detection, by themselves, were often not sufficient to segment such structures. Many methods have attempted to introduce prior knowledge of the shape of a structure into the object recognition process. A widely known technique, the "snake" or active contour method [8], used a deformable spline contour to capture the boundary of an object in an iterative energy minimizing process. The assumption of a smooth object boundary was guaranteed implicitly by the geometry of the spline contour. The level set method [12] was later proposed as a more powerful solution for handling instability and allowing changes in object topology. However, for objects with high curvatures or large boundary discontinuities, the smoothness assumption by itself is not sufficient for modeling object shape. Thus, some high-level prior knowledge is needed to guide the object segmentation process. Statistics based methods (e.g., [4]) used training data for recognizing objects with complicated 
shapes. Recently several methods (e.g., 11]) have incorporated shape priors into existing segmentation methods, e.g., [8 12], and presented promising results for applications in which closed contours can be used to model objects. For an object modeled by an open contour, Berger and Mohr's curve-growing method 2] can be applied. A shape model can also be used in the deformation of open contours, for example, as proposed by Akgual et al. [1].

A pulmonary fissure is a boundary between the lobes in the lungs. Its segmentation is of clinical interest because pulmonary nodules are frequently located adjacent to the fissure, whose identification would benefit computer aided diagnosis systems. Moreover, the ability to segment the lobes has additional clinical implications that include automated quantitative assessment of regional lung pathology and image registration. Few systems 91517 have addressed the problem of fissure segmentation. The main contributions of our paper are (1) an approach to include a shape prior in a curve-growing method for object segmentation (2) an adaptive regularization framework, (3) a process to address curve initialization and alleviate the local minima problem, (4) a successful application of the proposed method to the problem of segmenting fissures in CT. With the introduced shape prior, meaningful segmentation results are produced in the presence of uncertainties, such as ambiguous image features or high curvature variation on the object boundary. The adaptive regularization alleviates over-smoothing, an effect encountered by classical regularization methods. Our method also provides a solution for the initialization problem by taking advantage of the shape prior. It also alleviates the local minima problem effectively by a revised definition of the "image force."

\section{Method}

\subsection{Bayesian Formulation of Curve Growing}

Bayesian networks [13] have been applied to many applications that involve reasoning processes, as they succinctly describe causal dependencies using probabilities. Suppose that an object is modeled by a piecewise spline curve $\mathcal{C}$. A random variable $I$, representing the observed image data, and a random variable $\mathcal{C}^{*}$, representing prior information about the object shape, are considered as two causal predecessors of the random variable $\mathcal{C}$, the curve to be estimated. This relation can be modeled by the Bayesian network shown in Fig. 11A. The curve is represented as a collection of curve segments $\mathcal{C}=\left\{S_{1}, \ldots, S_{K}, \ldots, S_{N}\right\}$, where $S_{K}$ represents the $K$-th curve segment and is also considered a random variable. The curve $\mathcal{C}$ is created by adding curve segments $S_{K}$ one at a time. Random variable $S_{K}$ is assumed to be only dependent on the most recently added curve segment $S_{K-1}$ and not on earlier segments. We call this the "Markovianity assumption" [10] on subsequent curve segments. The corresponding Bayesian network is shown in Fig. 1B. Estimating the curve $\mathcal{C}$ is equivalent to finding the maximum of the joint probability $P\left(S_{1}, S_{2}, \ldots, S_{N}, \mathcal{C}^{*}, I\right)$ defined by the Bayesian network. By applying the Markovianity assumption, this is 


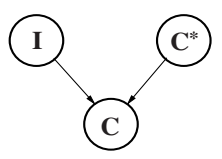

A

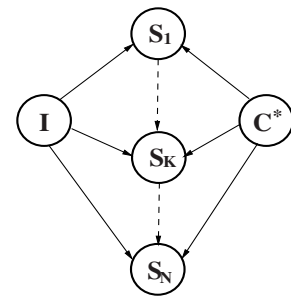

B

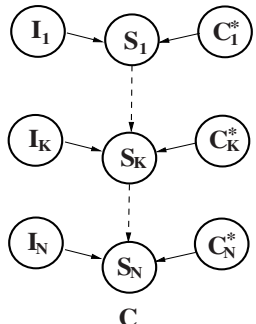

C

Fig. 1. A Hierarchy of Bayesian Networks for Curve Growing

$$
P\left(S_{1}, \ldots, S_{N}, \mathcal{C}^{*}, I\right)=P\left(\mathcal{C}^{*}, I\right) P\left(S_{1} \mid \mathcal{C}^{*} I\right) P\left(S_{2} \mid S_{1}, \mathcal{C}, I\right) \ldots P\left(S_{N} \mid S_{N-1}, \mathcal{C}^{*} I\right)
$$

This product includes the prior distribution $P\left(\mathcal{C}^{*}, I\right)$, which is generally assumed to be uniform, the posterior probability $P\left(S_{1} \mid \mathcal{C}^{*}, I\right)$, which models the probability of the initial curve placement and relates to the curve initialization problem, and the remaining posterior probabilities $P\left(S_{K} \mid S_{K-1}, \mathcal{C}^{*}, I\right)$, for $K=1, \ldots N$. The maximum of the joint probability given in Eq. 1 is approximated by the product of the maximum of $P\left(S_{1} \mid \mathcal{C}^{*}, I\right)$ and each $P\left(S_{K} \mid S_{K-1}, \mathcal{C}^{*}, I\right)$, where

$$
\begin{aligned}
& P\left(S_{K} \mid S_{K-1}, \mathcal{C}^{*}, I\right)=P\left(S_{K} \mid S_{K-1}, \mathcal{C}_{K}^{*}, I_{K}\right) \\
& =P\left(I_{K} \mid S_{K}, S_{K-1}\right) P\left(\mathcal{C}_{K}^{*} \mid S_{K}, S_{K-1}, I_{K}\right) P\left(S_{K}, S_{K-1}\right) / P\left(S_{K-1}, \mathcal{C}_{K}^{*}, I_{K}\right) .
\end{aligned}
$$

Here $I_{K}$ is the local image region containing $S_{K}$ and is considered to be the only relevant part of the image $I$ for estimating $S_{K}$ (Fig. 2). Similarly, $\mathcal{C}_{K}^{*}$ is the part of the shape prior $\mathcal{C}^{*}$ relevant to estimating $S_{K}$. The normalizing factor $P\left(S_{K-1}, \mathcal{C}_{K}^{*}, I_{K}\right)$ is considered irrelevant to $S_{K}$ and thus omitted. The corresponding Bayesian network is shown in Fig. 11C.

\subsection{Energy Function of Curve Growing Model}

The conditional probability $P\left(I_{K} \mid S_{K}, S_{K-1}\right)$ in Eq. 2 is defined as

$$
\mathbf{P}\left(I_{K} \mid S_{K}, S_{K-1}\right) \propto \exp \left(-\left|E_{i m g}\left(S_{K}\right)-E_{i m g}^{m i n}\right|\right)
$$

where $E_{i m g}\left(S_{K}\right)=-\sum_{i}\left|\nabla I\left(V_{K}^{i}\right)\right|$ is the associated image energy of curve segment $S_{K}, \nabla I$ defines the image force, in many applications, the intensity gradient, $V_{K}^{i}$ represents the $i$-th spline point in the segment $S_{K}$, and $E_{i m g}^{\min }$ is a lower bound on the values of $E_{i m g}$ that can occur in an image. In contrast to previous methods [816], the image energy is evaluated on the curve segment instead of a singular spline point, which reduces the possibility that the curve-growing process is trapped in off-curve local image energy minima.

In Eq. 2, the probability $P\left(\mathcal{C}_{K}^{*} \mid S_{K}, S_{K-1}, I_{K}\right)$ is used to model the similarity of the $K$ th segment of curve $C$ and prior curve $\mathcal{C}^{*}$. It is a function of $S_{K}, S_{K-1}$ and $I_{K}$, and can be defined by a Gaussian distribution: 


$$
\begin{gathered}
P\left(\mathcal{C}_{K}^{*} \mid S_{K}, S_{K-1}, I_{K}\right) \propto \exp \left(-\alpha\left(I_{K}\right) f_{\text {sim }}\left(S_{K}, S_{K-1}, C_{K}^{*}\right)\right) \\
=\exp \left(-\left|\mu\left(S_{K}, S_{K}^{*}\right)-\mu\left(S_{K-1}, S_{K-1}^{*}\right)\right| /\left(2 \sigma^{2}\right)\right) \text { with } \\
\mu\left(S_{K}, S_{K}^{*}\right)=\frac{1}{n} \sum_{i}\left|V_{K}^{i}-V_{K}^{i *}\right|, S_{K}^{*}=\left\{V_{K}^{i *} \mid V_{K}^{i} \in S_{K}\right\}, \text { and } C_{K}^{*}=S_{K}^{*} \cup S_{K-1}^{*},
\end{gathered}
$$

where $f_{\text {sim }}\left(S_{K}, S_{K-1}, C_{K}^{*}\right)$ is a function measuring the similarity between $\left(S_{K}\right.$, $\left.S_{K-1}\right)$ and the shape prior $C_{K}^{*},\left(V_{K}^{i}, V_{K}^{i *}\right)$ is a pair of corresponding points on $\mathcal{C}$ and $\mathcal{C}^{*}$ (correspondence is established by a closest-point search), $\mu\left(S_{K}, S_{K}^{*}\right)$ is the mean difference vector between two corresponding curve segments, $n$ is the number of points included in $S_{K}$, and $\alpha\left(I_{K}\right)$ is a function of $I_{K}$ used to control the magnitude of the Gaussian deviation $\left(\alpha\left(I_{K}\right) \propto 2 \sigma^{2}\right)$.

The smoothness constraint on the curve is modeled by:

$$
P\left(S_{K}, S_{K-1}\right) \propto \exp \left(-\left|f_{\text {curv }}\left(S_{K}, S_{K-1}\right)\right|\right),
$$

where $f_{\text {curv }}\left(S_{K}, S_{K-1}\right)$ is a function measuring the curvature between $S_{K-1}$ and $S_{K}$, e.g. as in Ref. [16]. By incorporating the Eqs. 35] into Eq. 2 and taking the logarithm, the curve energy function

$$
\begin{aligned}
& \mathbf{E}(\mathcal{C})=\mathbf{E}_{\text {shape }}(\mathcal{C})+\mathbf{E}_{\text {curv }}(\mathcal{C})+\mathbf{E}_{\text {img }}(\mathcal{C}) \propto \\
& \sum_{K=1}^{N}\left(\alpha\left(I_{K}\right) f_{\text {sim }}\left(S_{K}, S_{K-1}, C_{K}^{*}\right)+\beta_{K} f_{\text {curv }}\left(S_{K}, S_{K-1}\right)+\gamma_{K} E_{\text {img }}\left(S_{K}\right)\right)
\end{aligned}
$$

is obtained, where $\alpha, \beta$ and $\gamma$ regularization factors, whose ratios are constrained by the variances of the underlying Gaussian distributions. Each of $E_{\text {shape }}, E_{\text {curv }}$ and $E_{i m g}$ is normalized as suggested by Ref. [16]. The energy minimum of $\mathbf{E}(\mathcal{C})$ is considered an approximate MAP solution of the curve segmentation problem.

\subsection{Causal Confidence Approximation and Adaptive Regularization}

Ill-suited values for $\alpha, \beta$ and $\gamma$ in Eq. 6 can cause the curve $C$ to become overly smoothed. Regularization factors can be estimated by various approaches, for instance, a cross-validation method [10], which requires a large amount of offline computation and is then static during the segmentation phase. To obtain

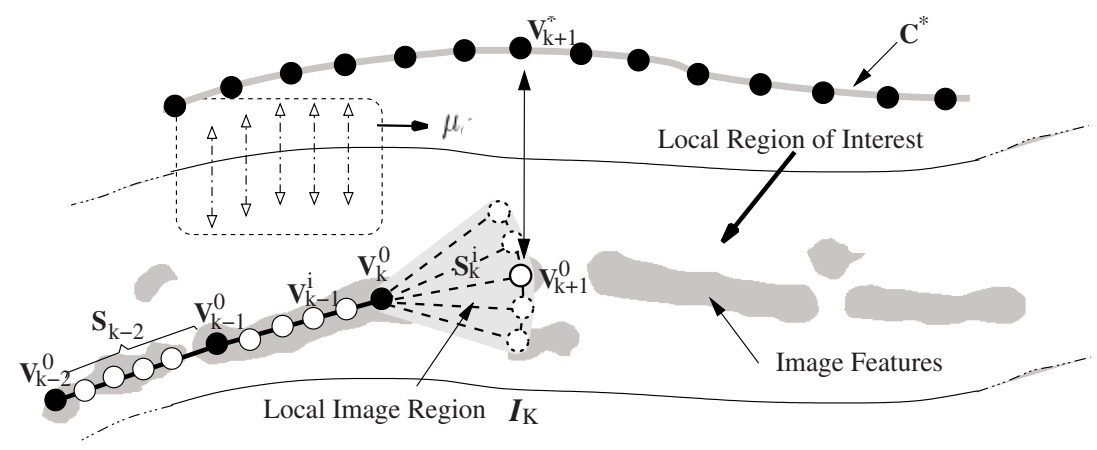

Fig. 2. Curve Growing with Adaptive Regularization 


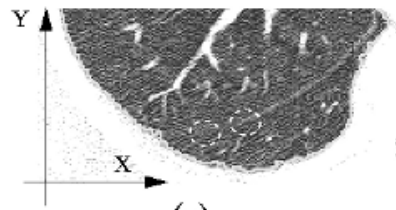

(a)

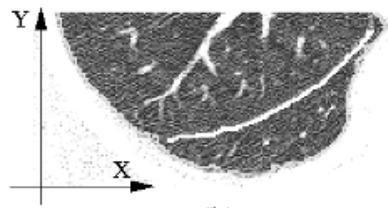

(b)

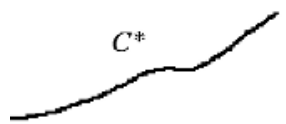

(c)

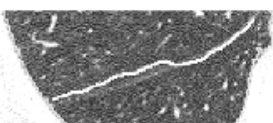

(f)

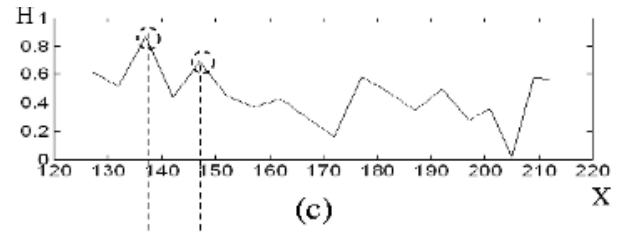

(c)

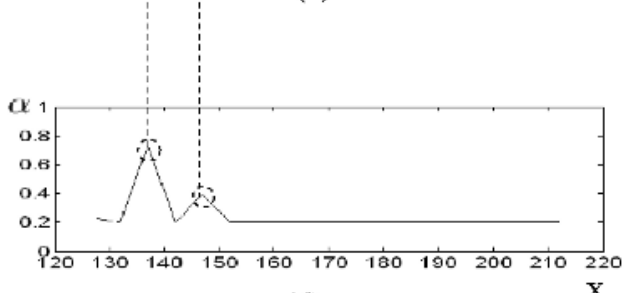

(d)

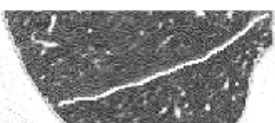

(g)

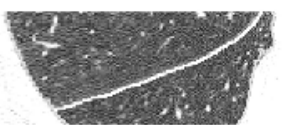

(h)

Fig. 3. Image features, entropy and adaptive regularization: Example 1: (a) Region of interest in a CT image. (b) Ground truth curve. (c) Entropy $H$ along the curve. (d) Corresponding value of $\alpha$. The two highest values of $H$ and $\alpha$ and the corresponding locations in the image are circled. Example 2: (e) Shape prior. (f) Curve obtained with static regularization, $\alpha=1.2$ and $\gamma=1.4$. (g) Curve obtained with adaptive regularization. (h) Ground truth curve.

a well-behaved regularizer, we propose an adaptive regularization framework. A revised factor $\alpha\left(I_{K}\right)$ is introduced to weigh adaptively the influence of image region $I_{K}$ versus the influence of prior shape $\mathcal{C}_{K}^{*}$. This also reflects the causal dependencies in the Bayesian network (Fig. 1 $\mathrm{k}$ ). The adaptive regularization parameter $\alpha\left(I_{K}\right)$ can be related to the entropy

$$
H\left(I_{K}\right)=-F \sum_{i=1}^{n} P\left(I_{K} \mid S_{K}^{i}, S_{K-1}\right) \log _{2} P\left(I_{K} \mid S_{K}^{i}, S_{K-1}\right)
$$

of image region $I_{K}$, where $S_{K}^{i}$ is the $i$-th sample of $S_{K}$ in $I_{K}$ (Fig. 2) and $F$ is a normalizing factor. The entropy $H\left(I_{K}\right)$ can be interpreted as the amount of uncertainty contained in image region $I_{K}$. For regions with large entropy values, the prior shape term should be weighted higher than for regions with small entropy values. The adaptive regularization parameter $\alpha\left(I_{K}\right)$ is thus defined as

$$
\alpha\left(I_{K}\right)=\max \left\{\epsilon,\left(H\left(I_{K}\right)-\lambda\right) /(1-\lambda)\right\},
$$

where $\lambda$ is the threshold that corresponds to a desirable feature in $I_{K}$ and can be learned offline from a set of training examples, and where $\epsilon$ defines the minimum value of $\alpha\left(I_{K}\right)$. The values $\lambda=0.5$ and $\epsilon=0.1$ were chosen in the current implementation. Fig. 3(a)- 3(e) gives an example where adaptive regularization satisfactorily strengthened the influence of the prior shape (circled areas). 


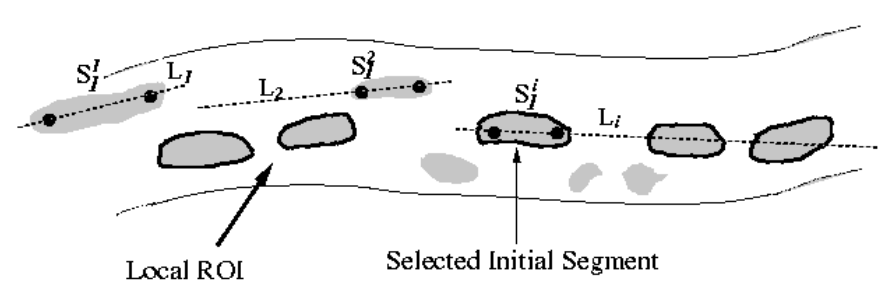

(a)

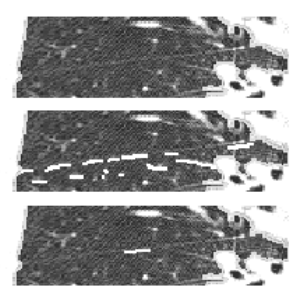

(b)

Fig. 4. Curve Initialization: (a) Alignments of relevant portions $\left\{L_{i}\right\}$ of prior curve $C^{*}$ with candidate segments $\left\{S_{1}^{i}\right\}$. (b) Local region of interest in CT (top), candidate segments $\left\{S_{1}^{i}\right\}$ (middle), and the selected initial curve segment $S_{1}$ (bottom).

Fig. 3(f)- 3(i) shows a second example, where adaptive regularization is clearly superior to static regularization.

\subsection{Curve Initialization}

The curve-growing process starts after a segment belonging to the curve is selected. The Bayesian formulation $P\left(S_{1} \mid \mathcal{C}^{*}, I\right)$ suggests that both the prior curve $C^{*}$ and the current image data $I$ should be considered in selection of $S_{1}$. In our work, the positions of salient image features, for example, local minima of the brightness gradient, are collected to form a set of candidate segments $\left\{S_{1}^{i}\right\}$. To choose $S_{1}$ among them, the prior curve $C^{*}$ is translated onto each $\left\{S_{1}^{i}\right\}$. A confidence weight is assigned to each candidate segment by accumulating image feature values along the relevant portion of translated $C^{*}$ (Fig. 4(a)). The segment with the highest confidence weight among all segments is chosen as the initial curve segment $S_{1}$. An example of the selection of an initial curve segment on CT is shown in Fig. 4(b). Our method provided more effective start conditions for the curve-growing process than Berger and Mohr's method [2] for most of our data.

\section{Results for Pulmonary Fissure Segmentation}

On CT, a fissure often looks like a ribbon structure with variable width due to respiratory motion and the partial volume effect. Fissures have been demonstrated as frequently incomplete on imaging [6] - they may appear as collections of discontinuous curve segments. Tissue surrounding the fissure, e.g., adjacent vessels or nodules, and clutter due to noise in the imaging process can result in off-curve local minima of the image energy. Traditional active contour methods 82 typically cannot overcome these difficulties.

The proposed method has been tested to segment pulmonary fissures on 11 thin-section CT scans of 4 patients. On each slice, a morphological operation was applied to generate a feature map of the local region of interest containing the fissure. The prior shape $C^{*}$ was estimated from fissures previously segmented in 


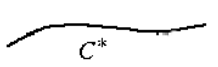

(a)

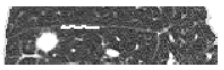

(b)

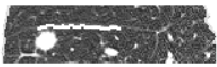

(c)

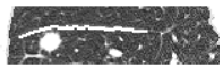

(d)

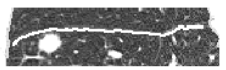

(e)

Fig. 5. Curve growing process: (a) Prior curve $C^{*}$. (b) Image region with initial curve segment $S_{1}$. (c)-(e) Intermediate results after 4,8 , and 18 iterations.
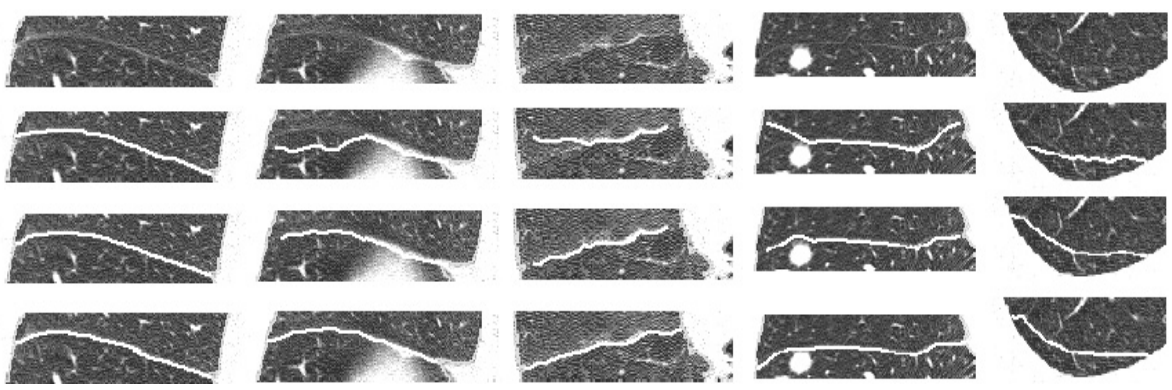

Fig. 6. Comparison of curve-growing results: Row 1: Original images. Row 2: Curve by method in Ref. 2]. Row 3: Curve by proposed method. Row 4: Ground-truth curve.
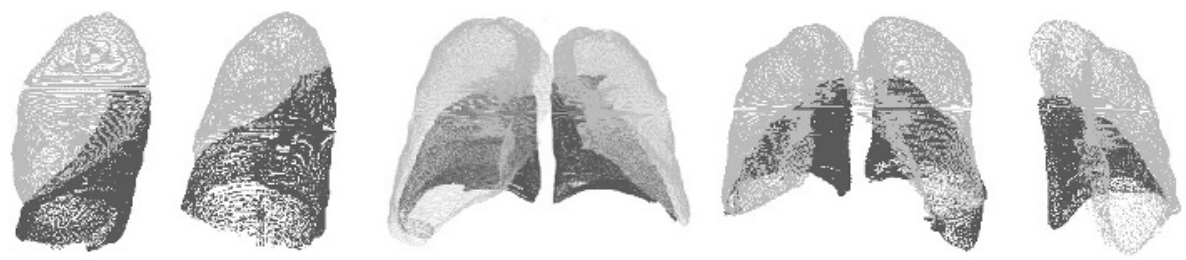

Fig. 7. Visualization of the segmented lung lobes of four patients.

other images of the same scan. For each CT scan, the fissure on a single slice was segmented semi-automatically; the fissures on the remaining slices were then segmented fully automatically (Figs. 5 and 6 ). The average time for segmenting fissures in one CT scan was less than 5 min on a PIII 1.2 GHz PC. Among 509 slices sampled from a total 1432 slices, the proposed method successfully segmented the fissures on 460 slices. The overall success rate was $460 / 509=90.4 \%$. The method then interpolated the segmentation of fissures on the remaining slices. Berger and Mohr's method [2] produced comparable results in the few cases where image features were sufficiently salient (Fig. 61). In many other cases, where image features were ambiguous, our method produced more meaningful results. Finally, the lung lobes were fully segmented by combining the segmented fissures with lung contours [3], as visualized in Fig. 7

\section{Conclusion and Future Work}

We described a shape-based curve-growing method and its application to pulmonary fissure segmentation. Effectively selecting the location of the next curve 
segment in each iteration of the curve-growing process is a critical part of our solution. It overcomes some of the difficulties encountered by other methods [8] 2. Moreover, the idea of adaptive regularization may be generalized and applied to other model-based energy-minimizing mechanisms. This will be investigated in future work involving different applications, possibly in higher dimensional spaces.

\section{References}

1. Y.S. Akgul, C. Kambhamettu, and M. Stone. Automatic extraction and tracking of the tongue contours. IEEE Trans Med Imag, 18:1035-1045, 1999.

2. M.O. Berger and R. Mohr. Towards autonomy in active contour models. In Tenth International Conference on Pattern Recognition, 847-851, Atlantic City, U.S., 1990.

3. M. Betke, J.B. Wang, and J.P. Ko. An integrated chest CT image analysis system - "BU-MIA." Tech. Report, Boston University, Computer Science Department, 2003.

4. T.F. Cootes, C.J. Taylor, D.H. Cooper and J. Graham. Active shape models their training and application. Comput Vis Image Underst, 61:38-59, 1995.

5. M.L. Giger and C.J. Vyborny. Computers aid diagnosis of breast abnormalities. Diagn Imaging, 15:98-102, 1993.

6. H.S. Glazer, D.J. Anderson, J.J. DiCroce, et al. Anatomy of the major fissure: evaluation with standard and thin-section CT. Radiology. 180:839-44, 1991

7. P. Golland, R. Kikinis, M. Halle, et al. AnatomyBrowser: A Novel Approach to Visualization and Integration of Medical Information. J Computer Assisted Surg, 4:129-143, 1999.

8. M. Kass, A. Witkin, and D. Terzopoulos. Snakes: Active contour models. Int J Comput Vis, 1:321-331, 1987.

9. M. Kubo, Y. Kawata, N. Niki et al. Automatic extraction of pulmonary fissures from multidetector-row CT images. In Proceedings of the IEEE International Conference on Image Processing. 1091-1094, Thessaloniki, Greece, 2001.

10. S.Z. Li. Markov Random Field Modeling in Computer Vision. Springer, 1995.

11. M.E. Leventon, W.E.L. Grimson, and O. Faugeras. Statistical shape influence in geodesic active contours. In Proceedings of the IEEE Conference on Computer Vision and Pattern Recognition, I:316-323, Hilton Head, U.S., 2000.

12. R.Malladi, J.A. Sethian and B.C. Vemuri. Shape modeling with front propagation: A level set approach. In IEEE Trans Pattern Anal Mach Intell, 17:158-175, 1995.

13. J. Pearl. Probabilistic reasoning in intelligent systems: Networks of plausible inference. San Mateo, California. Morgan Kaufmann, 1988.

14. D.L. Pham, C. Xu, and J.L. Prince. Current Methods in Medical Image Segmentation. Annual Review of Biomedical Engineering, 2:315+, 2000.

15. J. Wang, M. Betke, and J.P. Ko. Segmentation of pulmonary fissures on diagnostic CT - preliminary experience. In Proceedings of the International Conference on Diagnostic Imaging and Analysis, 107-112, Shanghai, China, 2002.

16. D.J. Williams and M. Shah. A fast algorithm for active contours and curvature estimation. CVGIP: Image Understanding, 55:14-26, 1992.

17. L. Zhang. Atlas-driven lung lobe segmentation in volumetric X-ray CT images. Ph.D. Thesis, University of Iowa. 2002. 